\title{
QUALITY IMPROVEMENT Engineering safer care coordination from hospital to home: lessons from the USA
}

\author{
Authors: Partha Das, ${ }^{\mathrm{A}, \mathrm{B}}$ James Benneyan, ${ }^{\mathrm{C}}$ Linda Powers, ${ }^{\mathrm{D}}$ Matthew Carmody, ${ }^{\mathrm{E}}$ Joanne Kerwin ${ }^{\mathrm{F}}$ and Sara Singer ${ }^{\mathrm{G}, \mathrm{H}}$
}

The safe transition of a patient from hospital into the community requires effective coordination between healthcare professionals across organisational boundaries. Preventing transition-associated failures can be especially challenging when multiple disciplines are involved and the patient has extensive care needs. The field of systems engineering is increasingly recognised as useful to help understand, improve and redesign such complex healthcare processes to improve patient experience and outcomes. To illustrate this approach, we describe how a partnership between healthcare professionals, systems engineers, and health services researchers used a series of engineering methods at a large suburban hospital to analyse and address deficiencies in a hospitalto-home transition process. Using this approach, the team designed a new process to perform more reliably despite inherent system complexity, demonstrating the value of systems engineering approaches and clinician-engineer collaborations.

KEYWORDS: Care transitions, systems engineering, quality improvement

\section{Introduction}

Close coordination of health and social care services is required for a patient to transition safely from hospital into

Authors: ${ }^{A}$ harkness fellow in healthcare policy and practice, Harvard TH Chan School of Public Health, Harvard University, Boston, Massachusetts, USA; ${ }^{B}$ harkness fellow in healthcare policy and practice, Healthcare Systems Engineering Institute, Northeastern University, Boston, Massachusetts, USA; ' professor of health systems engineering, Healthcare Systems Engineering Institute, Northeastern University, Boston, Massachusetts, USA; Dattending physician in internal medicine, Mount Auburn Hospital, Cambridge, Massachusetts, USA; ${ }^{E}$ attending physician in internal medicine, Mount Auburn Hospital, Cambridge, Massachusetts, USA; ${ }^{\mathrm{F}}$ director of quality and performance, CareGroup Parmenter Home Care and Hospice, Watertown, Massachusetts, USA; ${ }^{G}$ professor of health policy and management, Harvard TH Chan School of Public Health, Harvard University, Boston, Massachusetts, USA; ${ }^{H}$ professor of medicine, School of Medicine, Stanford University, Stanford, California, USA the community. Problems arise when this process becomes fragmented or falters in some way. The incidence of delayed transfers of care per year arising from ineffective care coordination in England is increasing."

Ramifications of less-effective care transitions include poorer patient experience, lower quality of life, greater adverse events, and higher risks of readmission to hospital with an itinerant increased risk of death. ${ }^{2-6}$ The annual financial cost to the NHS of hospital readmissions from care transition-associated failures is estimated to be $£ 2.4$ billion. ${ }^{3}$

Quality improvement (QI) approaches to address this problem include the Institute for Healthcare Improvement (IHI) Model for Improvement, methods derived from manufacturing such as Six Sigma and Lean, and, more recently, systems engineering. ${ }^{7-9}$

Systems engineering focuses on different but complementary methods to traditional QI to understand, improve, design, and integrate complex interdependent systems. In 2015 the USAbased Agency for Healthcare Research and Quality (AHRQ) funded a series of patient safety learning laboratories (PSLLs) to explore the benefits of systems engineering and systems thinking to a number of challenging and persistent healthcare problems.

One of 13 PSLL initiatives, the Engineering High Reliability Learning Lab (EHRLL) in Boston Massachusetts is a collaboration of four healthcare systems and two universities focused on applying these approaches to achieve better care coordination across organisational boundaries. One EHRLL project at Mount

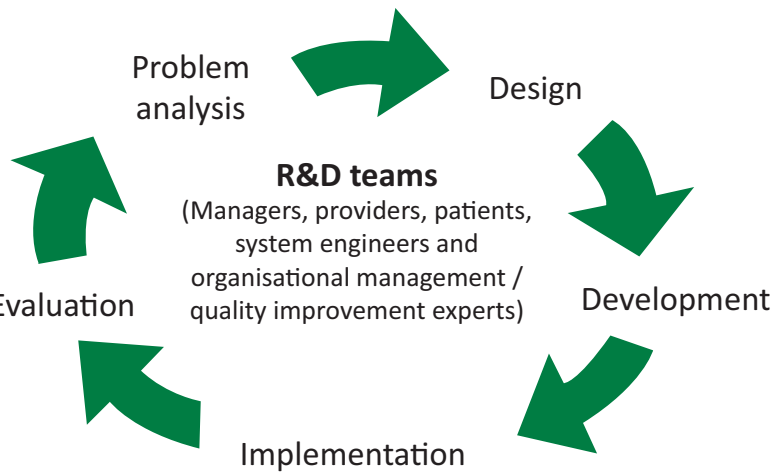

Fig 1. Five phases of the EHRLL innovation cycle and membership of the teams involved at each clinical site. EHRLL = Engineering High Reliability Learning Lab; R\&D = research and development 


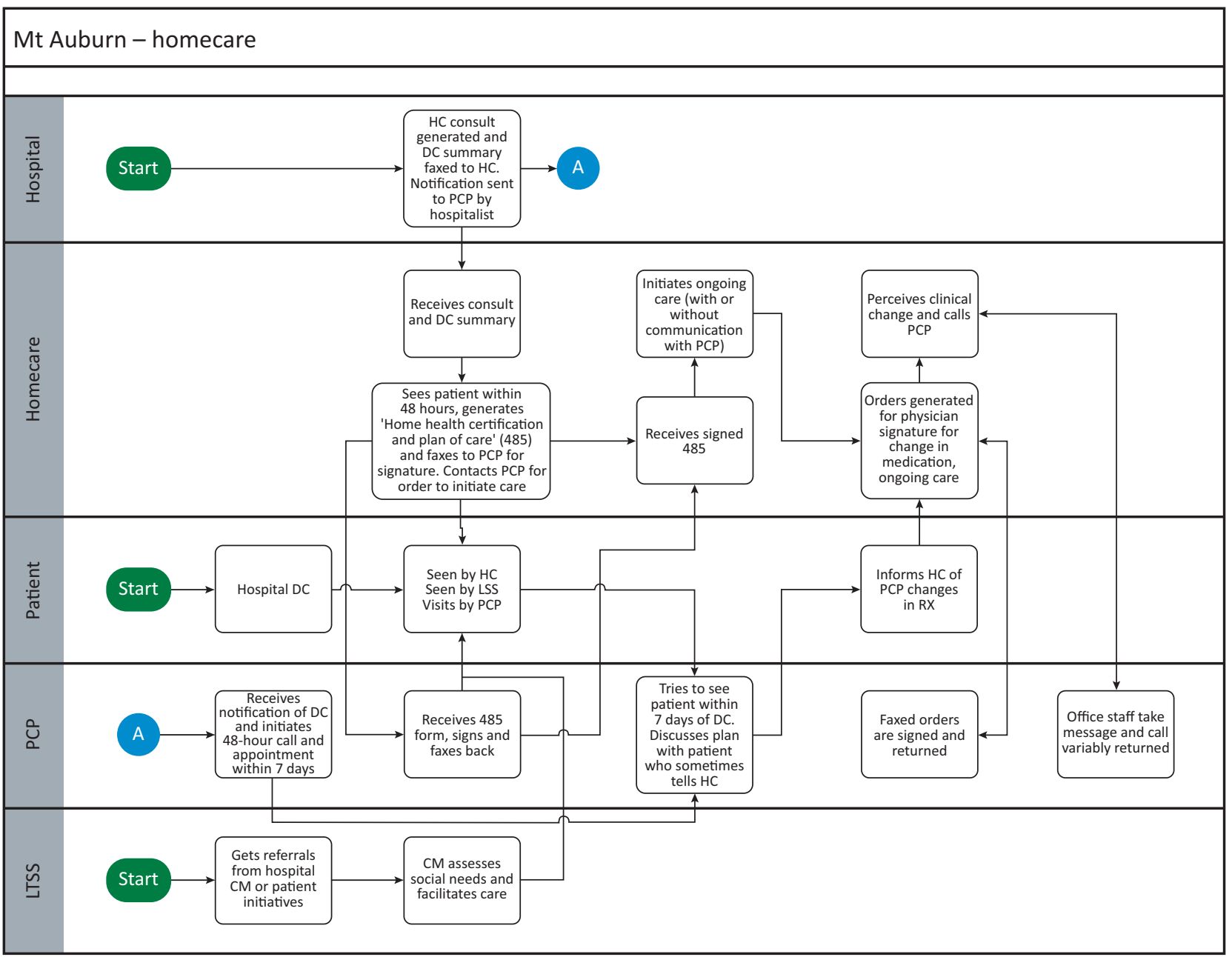

Fig 2. Swim lane diagram outlining steps within the discharge process and separated by participant showing points of interaction. Initial discharge process. $\mathrm{DC}=$ discharge; $\mathrm{HC}$ = homecare; hospitalist = lead physician in hospital; $\mathrm{PCP}$ = primary care physician; LTSS = long-term services and support (includes social worker, behavioural health specialist, physical therapist); $\mathrm{RX}=$ medication prescription

Table 1. Failure modes effect analysis (FMEA). Each failure mode is scored for severity (sev), probability of occurrence (prob) and detectability (det) by the system which are multiplied to give the risk priority number (RPN) that ranks the failure modes. The higher the RPN, the worse the failure mode.

\begin{tabular}{|c|c|c|c|c|c|}
\hline & & & & & \\
\hline $\begin{array}{l}\text { Failure } \\
\text { number }\end{array}$ & Failure & $\overline{\operatorname{Sev}}$ & Prob & Det & RPN \\
\hline 1 & $\begin{array}{l}\text { No mechanism of direct communication between homecare, hospitalist, PCPs about high- } \\
\text { risk, complex patients }\end{array}$ & 9 & 9 & 7 & 567 \\
\hline 2 & Content of communication between homecare, hospitalists, PCPs is not accurate/complete. & 7 & 8 & 1 & 56 \\
\hline 3 & Inpatient and outpatient care do not work together as a team & 9 & 9 & 10 & 810 \\
\hline 4 & No standard agreement on risk identification / the underestimation of risk & 9 & 8 & 9 & 648 \\
\hline 5 & Lack of common understanding of who does what when & 7 & 8 & 8 & 448 \\
\hline 6 & Medication reconciliation - lack of understanding about what it is, when it should happen & 9 & 8 & 9 & 648 \\
\hline 7 & Learning curve for the patient in the hospital & 9 & 7 & 10 & 630 \\
\hline 8 & Lack of assessment of patient / caregiver skills / abilities & 8 & 8 & 6 & 384 \\
\hline 9 & Lack of patient engagement / underappreciation of patient needs & 7 & 8 & 4 & 224 \\
\hline 10 & Patient doesn't know who to call (PCP or homecare) under varying circumstances & 6 & 4 & 10 & 240 \\
\hline 11 & Patient preferences different to caregiver perception & 8 & 10 & 4 & 320 \\
\hline 12 & Staff resources overwhelmed & 9 & 7 & 6 & 378 \\
\hline 13 & Patient at risk between their arrival home and homecare nurse arrival & 8 & 7 & 6 & 336 \\
\hline
\end{tabular}




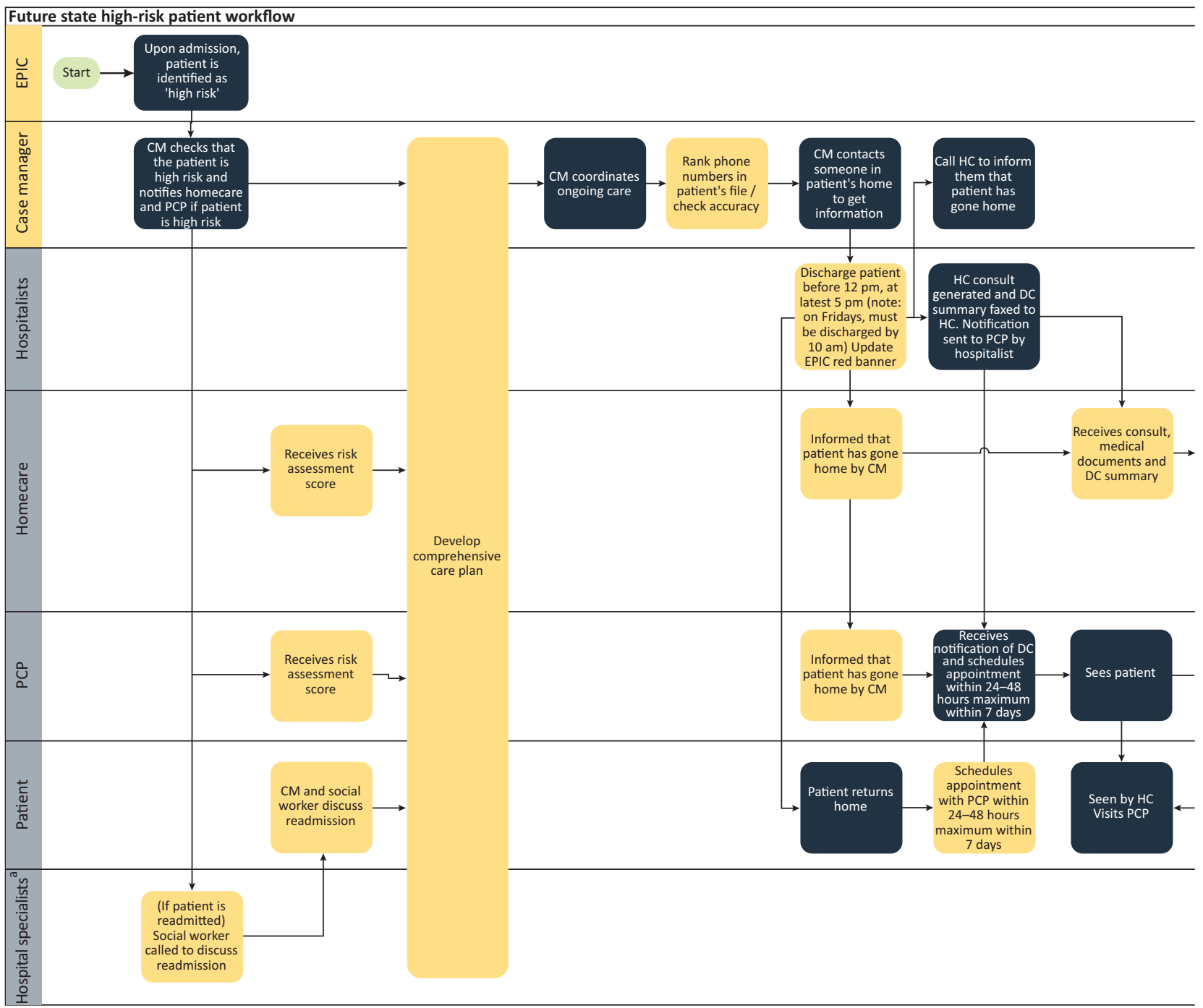

Fig 3. Swim lane diagram outlining steps within the discharge process and separated by participant showing points of interaction. Redesigned discharge process with identification of high risk patient within electronic health record (EPIC) and new swim-lanes for case manager (CM) and allied health care professionals (hospital specialists). DC = discharge; HC = home-care; hospitalist = lead physician in hospital; $\mathrm{PCP}=$ primary care physician; $\mathrm{LTSS}=$ long-term services and support

Table 2. Extract of contrast analysis comparing common themes influencing the care of a high-risk patient who was not readmitted after discharge with a low-risk patient who was readmitted

\section{Success}

Patient

Diagnosis

Hospitalisations

Result

Factors
Male, $>90$ years old

Heart disease, renal failure, cardiac cachexia

5 ( 4 in first 6 months)

Patient lived 9 months past prognosis and passed away at home

$>$ Family member as sole care coordinator, lived in

> 3.6 clinic visits per months

> Responsive, well-connected homecare nurse(s) consistently updated PCP

\section{Failure}

Female, between 80 and 90 years old

Progressive dysphagia from pharyngeal cancer

1

Patient admitted to SNF after 6 days at home

Incorrectly assessed that patient / homecare could manage g-tube

> Case management, PCP and homecare failed to find acceptable solution

$\mathrm{G}$-tube = gastrostomy tube; $\mathrm{PCP}=$ primary care physician; $\mathrm{SNF}=$ skilled nursing facility 


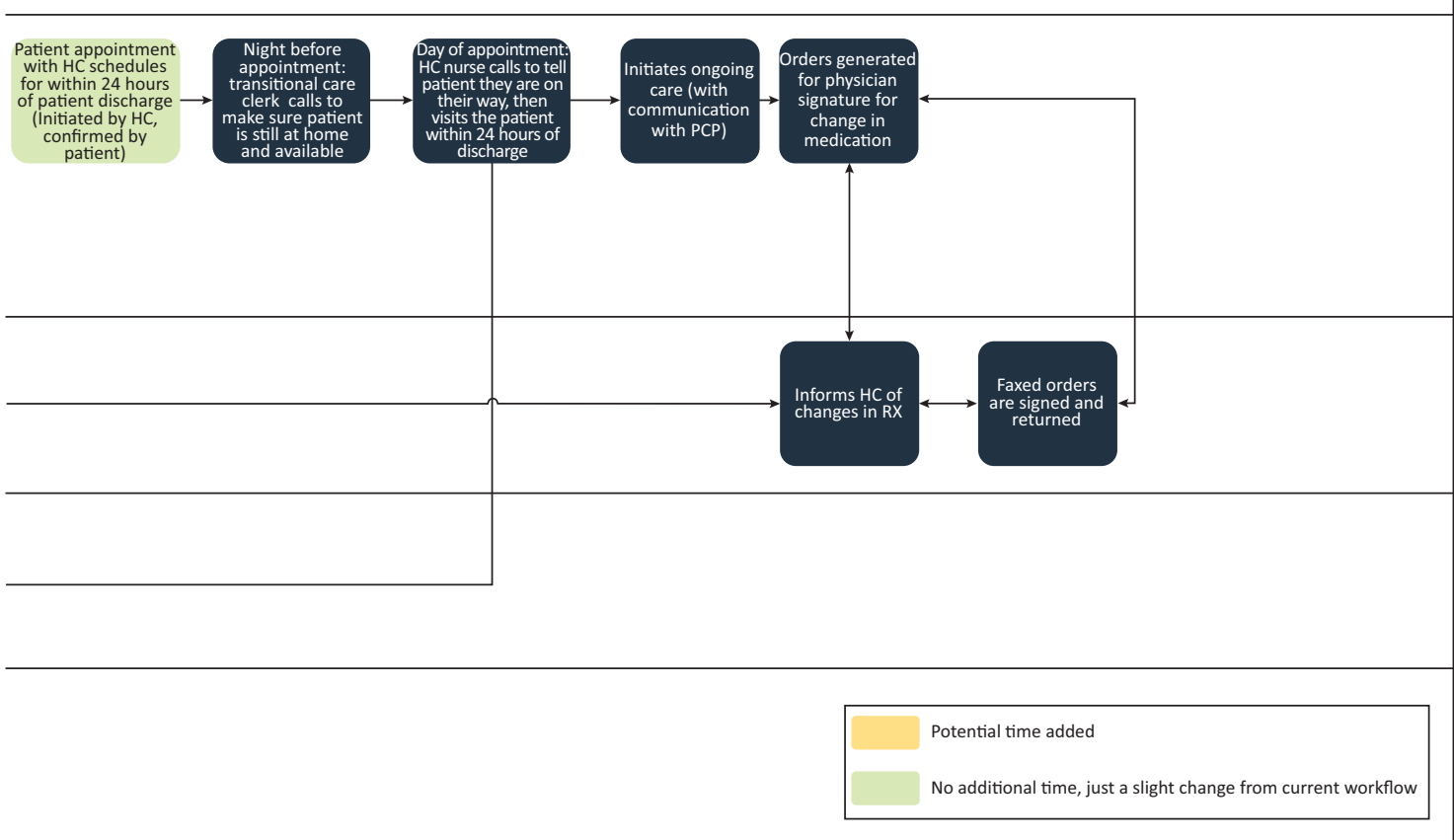

Auburn Hospital (MAH) is focusing on high-risk patient discharges from hospital to home. MAH is a 213-bed suburban hospital in Cambridge, Massachusetts with nearly 9,000 patient discharges in 2017. The EHRLL team partnered with MAH to design a new process to improve patient experience, reduce post-discharge adverse events, foster community home-based care, and reduce emergency readmissions as well as costs.

\section{Methodology}

An interdisciplinary team of MAH clinicians, hospital and community care leadership, systems engineers from the Healthcare Systems Engineering Institute at Northeastern University, and healthcare organisational management and QI researchers from Harvard University (public health and medical schools) was formed. Further input to the team was sought as needed from therapies staff and caregivers, community services, and patient representatives. The MAH team met weekly to apply
QI and systems engineering methods, supported by engineering students and triannual half-day meetings of all EHRLL teams for sharing, reflecting, and feedback. In parallel, the Harvard researchers used mixed methods to prospectively study team and meeting dynamics, barriers limiting the use of engineering methods, constraints hindering problem-solving, and successful strategies.

A systems engineering lifecycle was followed to guide the sequence of work and methods used (Fig 1). The lifecycle starts with a problem analysis phase, a design phase where requirements for new processes are established based on problem analysis, and a development phase where the most suitable design ideas are prototyped via a rapid testing and evaluation process similar to plando-study-act (PDSA) cycles. An implementation phase then tests the redesigned process in a wider context before undergoing an impact assessment in the evaluation phase. In practice, this cycle repeated itself as the team addressed different aspects of the process. 
Within this framework, systems engineering approaches were used to:

$>$ understand existing processes for hospital-to-home transitions

> learn from successes and failures within this existing system

> facilitate system redesign to create a new and more reliable care transition process.

Specific systems engineering methods included cross-functional process maps ('swim-lane diagrams'), failure modes and effects analysis (FMEA), contrast analysis, and the functional resonance analysis method (FRAM). Since many of these methods were unfamiliar to the healthcare professionals on the team, coaching and facilitation in their use was provided by the embedded engineers.

\section{Results}

\section{Understanding existing processes for hospital-to-home} transition

Process mapping allows the visualisation of a care pathway and is a feature of several QI methods. ${ }^{10}$ The team developed an initial process map of the transition to home process, which the engineers then iteratively revised into a cross-functional swim lane diagram (Fig 2). This schematic describes the specific sequence of steps during patient discharge from hospital into the community with the roles of each participant delineated within 'swim lanes' and with interactions shown as crossovers between lanes.

The swim lane diagram showed that during discharge, contact was delayed, infrequent, or inadequate in content between responsible healthcare professionals within the hospital and in the community. Communication of some critical pieces of information relied on transmission by fax, increasing the possibility of information loss or delay. For example, timely post-discharge primary care physician review of the patient was dependent on community care visiting the patient within 48 hours of discharge, having access to all relevant information pertinent to the admission, and faxing an ongoing care plan back to a physician for review at a particular point in time. The latter was problematic because the care plan was inevitably a continuously evolving document. Furthermore, it became apparent during the development of the swim lane that many antecedents of adverse patient outcomes in the community occurred early in the hospital stay, expanding the team's focus to include this part of the patient journey.

\section{Learning from success and failure within the existing pathway}

Concepts from patient safety were drawn upon during the problem analysis phase, blending traditional and new methods from both the 'Safety-I' and 'Safety-II' fields. ${ }^{11}$ Safety-I typically seeks linear causes of failures within a system using tools such as root cause analysis (RCA). The team used failure modes and effects analysis (FMEA), ${ }^{12,13}$ a common engineering method that engages key stakeholders in brainstorming and prioritising potential 'failure modes' or ways in which the hospital-to-home discharge process can fail. These identified failure modes were scored by each team member in three domains: a) the severity of the failure if it occurs, b) the probability of that failure occurring, and c) the inability of the system to detect and mitigate the failure if it occurs. An overall risk priority number (RPN) for each type of failure then was calculated by multiplying these severity, probability, and detectability scores, with the ranked results being used to prioritise process redesign efforts (Table 1). The FMEA methodology helped the team systematically prioritise failure modes by focusing on those with the highest RPN values.

The top three failure modes for the MAH project were related to

1 a lack of teamwork across hospital and community care

2 no standard method for early identification of patients at high risk of rehospitalisation

3 incomplete medication reconciliation both before and after being discharged home.

Despite the clear utility of RCA and FMEA methods, each can be limited by their intrinsic inability to deal with non-linear causality and complexity. ${ }^{14}$

Safety-II principles in contrast assume much less of a linear 'cause and effect' manner of thinking. Instead there is the view that complex systems themselves, despite their inherent variability, do not fail most of the time and that often the same conditions and root causes produce both safe and unsafe events. To capture an aspect of 'normal' functioning within the system, the team therefore performed a contrast analysis (Table 2) to compare an 'extreme success' case (a high-risk patient who was not readmitted after discharge) with an 'extreme failure' case (a low-risk patient who was readmitted nonetheless). Referencing each process step from the swim lane diagram, a key finding from this exercise was that 'successful' patient journeys often featured frequent and structured communication between the responsible clinician and community nurse, inherently mitigating some of the same high-risk failure modes identified by the FMEA.

\section{Facilitating system redesign to create a new transition process}

The FMEA and contrast analysis together enabled the team to identify a set of primary drivers to underpin a redesigned care transition process for higher risk patients. A second swim lane diagram was constructed to depict a new idealised design, with three key differences from the original process including a) an initial readmission risk assessment at hospital admission, b) designated protocols for communication between inpatient and community team members at critical junctures, and c) a shared multidisciplinary care plan (Fig 3). As an example of a less familiar engineering design tool, FRAM ${ }^{15}$ was used to design necessary details of the new process. This method helped the team to visualise how each activity or 'function' in the revised process relates to each other and to describe each function by its needed inputs, outputs, time, resource, preconditions, and controls. When failures occur in complex systems, the individual interconnected functions can have unpredictable outputs and variability that are either absorbed resiliently or aggregate to reinforce each other, 'resonating' to produce the type of extreme successes or failures explored in the contrast analysis (Fig 4).

\section{Conclusion and next steps}

The issues facing Mount Auburn Hospital and their community partners will be familiar to organisations in the UK and highlight the importance of closer collaboration between the NHS and social care. For newer models of care such as those described 


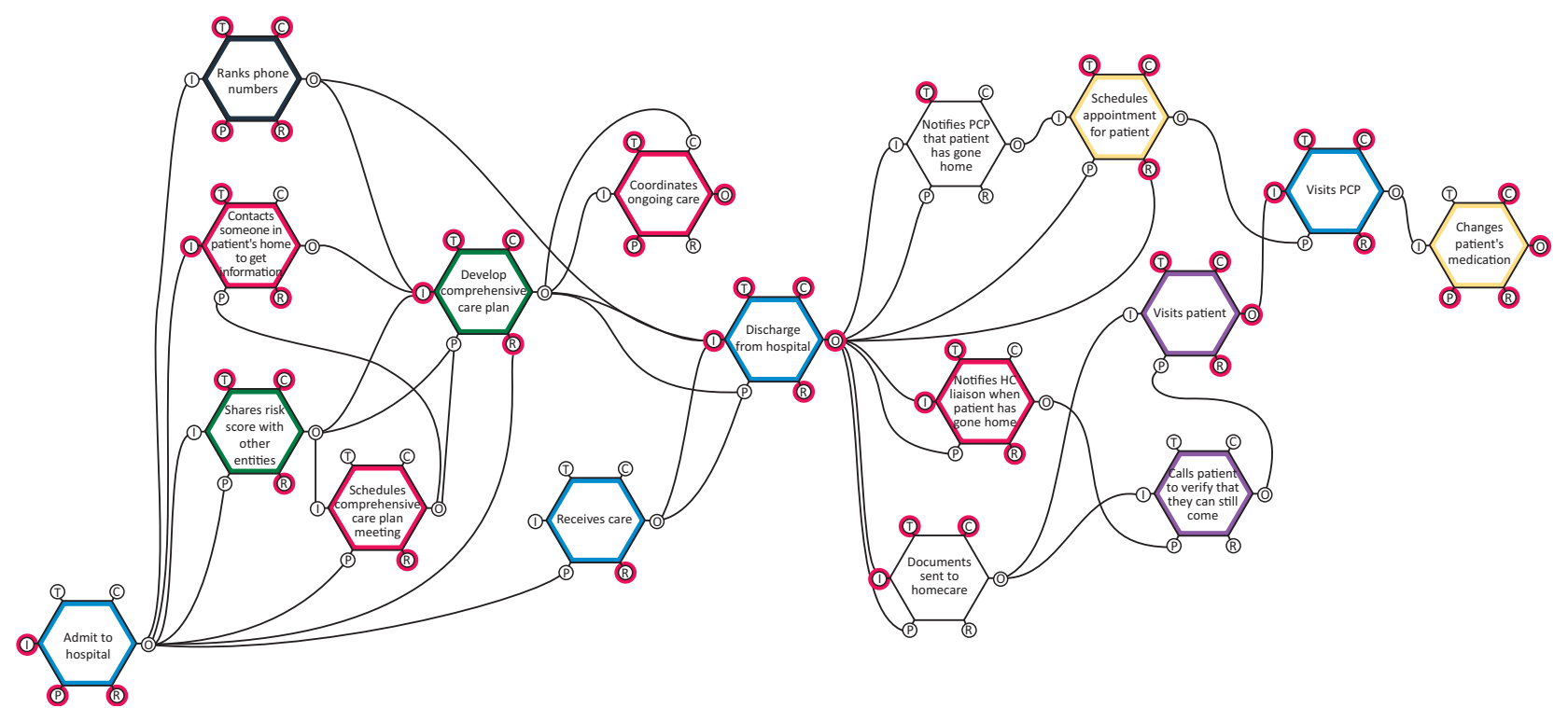

Fig 4. Functional resonance analysis method (FRAM) diagram. Each 'function' (hexagon) corresponds to a step in the revised discharge process (Fig 3) and shows how functions can be both inputs (I) and outputs ( $\mathrm{O}$ ) in the system and also considers how other factors (time [T], resource [R], preconditions [P] and control inputs [C]) influence each function. Important factors for each function are highlighted in red circles. Different coloured hexagons correspond to a different participant or group of participants in the process. The FRAM approach allowed the team to better understand system complexity and visualize how functions relate to each other. Hexagon colours: blue = patient; black (thick) = electronic medical record; pink = case manager; yellow = primary care physician; purple = homecare; green = all participants; black (thin) = clerical support.

in regional Sustainability and Transformation Partnerships (STPs - multiple health and social care organisations within 44 regions in England collaborating together with the aim of providing better coordinated care for their local population) to be successful, innovative approaches that work within the complexity of multiple services interacting together are required ${ }^{16}$ rather than limited focus projects that might fail due to underappreciation of complex cause and effect. ${ }^{17}$ The field of systems engineering is ideally suited for addressing these types of complex problems and provides a repertoire of methods and concepts that can help solve difficult healthcare problems.

To accomplish this potential, most publications advocating greater application of systems engineering in healthcare include recommendations to cultivate clinician-engineer partnerships and interdisciplinary collaborations. EHRLL has demonstrated one viable approach for partnering clinicians and engineers and for effectively deploying systems engineering methods. Using the engineering design lifecycle and a range of tools, the $\mathrm{MAH}$ team developed a deeper insight into the interdependent processes within which the inpatient, outpatient and community personnel work, the roles of their fellow healthcare professionals, and alignment with patient preferences beyond that recognised through QI approaches alone. Ongoing work of the MAH team includes operationalising and refining the new process design, measuring its impact quantitatively and qualitatively, and tracking several process and outcome measures over time. As a shorter-term surrogate measure, and to help build will for change, a consensus-based rescoring of the FMEA suggests that once fully implemented this work will result in a $77 \%$ reduction in total patient safety risk (RPN), including a $86 \%$ reduction in the top four failure modes.

The healthcare professionals involved in this project had to appreciate that their approach to problem-solving was distinct to the engineers and vice versa, requiring both groups to think differently. Although use of engineering methods unfamiliar to the healthcare team required a significant time investment in both training and application, this helped strengthen relationships between the healthcare teams and engineering personnel. The facilitated application of engineering methods such as process maps, failure analysis, and design thinking also contributed to better team-work, identified knowledge gaps, and resulted in new process designs beyond simply minor tweaks to existing work flows.

The Royal College of Physicians and The Royal Academy of Engineers white paper Engineering Better Care proposes a useful role for systems engineering in the NHS. ${ }^{7}$ Our experience at MAH reinforces this potential utility and demonstrates one way of deploying systems engineering in healthcare settings. To leverage this potential more broadly, an important logistical consideration is how a pipeline of systems engineering talent might be cultivated and how this might function alongside recent NHS trust investments to develop QI capability. ${ }^{18}$ Beyond EHRLL, it is clear from years of similar experiences that to scale systems approaches will require greater training of clinical teams in systems engineering principles and greater partnering of engineers with healthcare improvement teams, ${ }^{19}$ although the value of doing so is equally clear.

\section{References}

1 NHS England. Delayed transfers of care statistics for England. NHS England, 2017.

2 Moore C, Wisnivesky J, Williams S et al. Medical errors related to discontinuity of care from an inpatient to an outpatient setting. J Gen Intern Med 2003;18:646-51.

3 Healthwatch England. Safely Home: What happens when people leave hospital and care settings? Healthwatch England, 2015.

4 Forster AJ, Clark HD, Menard A et al. Adverse events among medical patients after discharge from hospital. CMAJ 2004;170:345-9. 
5 Balaban RB, Weissman JS, Samuel PA et al. Redefining and redesigning hospital discharge to enhance patient care: a randomized controlled study. J Gen Intern Med 2008;23:1228-33.

6 Gonçalves-Bradley DC, Lannin NA et al. Discharge planning from hospital. Cochrane Database Syst Rev 2016:CD000313.

7 Clarkson PJ, Bogle D, Dean J et al. Engineering better care: A systems approach to health and care design and continuous improvement. London: Royal Academy of Engineering, 2017.

8 Watts BV, Shiner B, Ceyhan ME et al. Health systems engineering as an improvement strategy: a case example using location-allocation modeling. J Healthc Qual 2013;35:35-40.

9 National Academy of Engineering. Building a better delivery system: A new engineering/health care partnership. Reid PP, Compton WD, Grossman JH, Fanjiang G (eds). Washington, DC: The National Academies Press, 2005.

10 NHS Improvement. Process mapping - a conventional model. NHS Improvement, 2018.

11 Hollnagel E, Wears RL, Braithwaite J. From Safety-I to Safety-II: A White Paper. The Resilient Health Care Net: Published simultaneously by the University of Southern Denmark, University of Florida, USA, and Macquarie University, Australia, 2015.

12 DeRosier J, Stalhandske E, Bagian JP, Nudell T. Using health care failure mode and effect analysis: the VA National Center for Patient Safety's prospective risk analysis system. Jt Comm J Qual Improv 2002;28:248-67.
13 Goodrum L, Varkey P. Prevention is better: the case of the underutilized failure mode effect analysis in patient safety. Isr ] Health Policy Res 2017;6:10.

14 Peerally MF, Carr S, Waring J, Dixon-Woods M. The problem with root cause analysis. BMJ Qual Saf 2017;26:417-22.

15 Hollnagel E. FRAM, the functional resonance analysis method: modelling complex socio-technical systems. Ashgate, 2012.

16 Braithwaite J. Changing how we think about healthcare improvement. BMJ 2018;361:k2014.

17 Charles A, Ham C, Baird B, Alderwick H, Bennett L. Reimagining community services: Making the most of our assets. The King's Fund, 2018.

18 NHS Improvement. Building capacity and capability for improvement: embedding quality improvement skills in NHS providers. NHS Improvement, 2017.

19 Benneyan J, Balint C. Improving healthcare by teaming industrial engineers with clinicians. ASEE Annu Conf Expo Conf Proc, 2015

Address for correspondence: Dr Partha Das, Harvard TH Chan School of Public Health, Health Policy and Management, 677 Huntington Avenue, Boston, Massachusetts 02115, USA. Email: parthadas@doctors.net.uk 